\title{
A novel method for obtaining highly enriched Schwann cell populations from mature monkey nerves based on in vitro pre-degeneration
}

\author{
GANGYANG WANG $^{1 *}$, ZHENGWEN MA $^{2 *}$, LINGLING CAO ${ }^{3 *}$, GUOFENG YAN $^{2}$, YANG WANG $^{1}$,

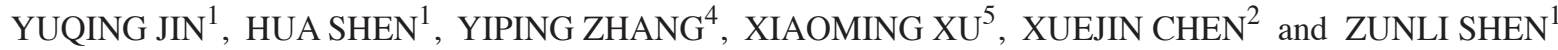

\begin{abstract}
${ }^{1}$ Department of Plastic and Reconstructive Surgery, Shanghai General Hospital, Shanghai Jiao Tong University School of Medicine, Shanghai 200080; ${ }^{2}$ Department of Laboratory Animal Science, Shanghai Jiao Tong University School of Medicine, Shanghai 200025; ${ }^{3}$ Department of Rehabilitation Medicine, Shanghai General Hospital, Shanghai Jiao Tong University School of Medicine, Shanghai 200080, P.R. China; ${ }^{4}$ Norton Neuroscience Institute, Norton Healthcare, Louisville, KY 40202;

${ }^{5}$ Spinal Cord and Brain Injury Research Group, Stark Neurosciences Research Institute, Department of Neurological Surgery, Indiana University School of Medicine, Indianapolis, IN 46202, USA
\end{abstract}

Received November 12, 2016; Accepted July 12, 2017

DOI: $10.3892 / \mathrm{mmr} .2017 .7427$

\begin{abstract}
Schwann cells (SCs) are indispensable for cell therapy and tissue engineering of the peripheral nervous system. Easy access to activated, highly proliferative SCs is necessary for clinical applications. The present study developed a fast, efficient method for obtaining highly purified SCs from the peripheral nerve of a mature Rhesus monkey. The common peroneal nerves of 4-year-old Rhesus monkeys were harvested and subjected to in vitro pre-degeneration in a modified SC culture medium (SCCM). The nerve pieces were subsequently treated enzymatically to dissociate the cells and then cultured for 2 days in SCCM. Cultured cells were treated with purification medium containing Ara-C to assist in restricting the overgrowth of fibroblast-like cells, for $24 \mathrm{~h}$. After another 24-h cultivation period, the cells were subsequently treated with a multiplex collagenase, which enabled SC detachment over fibroblast detachment, and thereby facilitated SC isolation. Finally, SCs were cultured in SCCM. The cell yield was determined by cell counting following enzyme
\end{abstract}

Correspondence to: Dr Zunli Shen, Department of Plastic and Reconstructive Surgery, Shanghai General Hospital, Shanghai Jiao Tong University School of Medicine, 100 Haining Road, Shanghai 200080, P.R. China

E-mail: zunlishen@163.com

Dr Xuejin Chen, Department of Laboratory Animal Science, Shanghai Jiao Tong University School of Medicine, 227 Chongqing Road, Shanghai 200025, P.R. China

E-mail: chenxuej@aliyun.com

${ }^{*}$ Contributed equally

Key words: Schwann cells, monkey, pre-degeneration, nerve regeneration, cell culture digestion and SC purity was determined from the percentage of SCs with respect to the total number of cells. Following purification, $96.3 \pm 3.9 \%$ of cells were identified as SCs. In vitro pre-degeneration in the presence of basic-fibroblast growth factor, heregulin $\beta 1$ and forskolin maximized the purity and yield of SCs that could be obtained from monkey peroneal nerves. The present study identified a novel technique that can efficiently isolate and purify SCs from mature monkey nerves based on in vitro pre-degeneration.

\section{Introduction}

Schwann cells (SCs) are the predominant glial cells in the peripheral nervous system and serve a vital role in the growth and regeneration of peripheral nerves (1). Following nerve injury, the denervated SCs begin to dedifferentiate, proliferate and migrate, and subsequently form linear arrays in the original endoneurial tubes termed bands of Büngner; these arrays provide a remote channel for axon regeneration (2). Previous studies have indicated that nerve regenerative potential may be enhanced if cells, particularly SCs, cover the bio-artificial tube $(3,4)$. The complex of scaffolding and SCs acts as the core of tissue-engineered nerves and SCs are the most important seed cells of tissue-engineered nerves (5). Therefore, developing an efficient method of SC culture is essential for the success of nerve repair techniques.

The major obstacle to SC purification is the removal of fibroblasts. Numerous fibroblast removal strategies have been reported including serum-free medium treatment (6), anti-mitotic treatment (7), immunoselective methods (8) and differential detachment methods (9), amongst others. Another method is based on pre-degeneration following peripheral nerve injury (10). Pre-degeneration may be performed in vivo and in vitro. In vivo (compared with in vitro) pre-degeneration is more cumbersome to perform as it requires two surgical interventions and takes longer to accomplish (11). This method is not applicable to humans for ethical reasons; 
therefore, the present study focused on developing an in vitro pre-degeneration method. During in vitro pre-degeneration, harvested nerve pieces were placed in a specialized medium prior to enzymatic digestion. The purpose of this procedure was to stimulate the proliferation of SCs, and to promote fibroblast migration from the nerve pieces. A previous study has indicated that, compared with immediate culture, performing in vitro pre-degeneration prior to cell culture of the harvested cells may increase SC purity and yield (11). The conditions of pre-degeneration may impact the purity and yield of cultivated SCs (12). Kraus et al (13) demonstrated that in vitro pre-degeneration for 7 days increased the yield of SCs by $\sim 50 \%$; however, different periods of pre-degeneration had limited effect on the purity of the SCs. Furthermore, pituitary extracts (14) and neuregulins (15) were shown to stimulate SC proliferation.

Based on previous experience using multiple factors as SC proliferation promoters (16), basic fibroblast growth factor (b-FGF), heregulin and forskolin were selected to aid nerve pre-degeneration and SC culture, which was performed over a 7-day period. The present study reported a novel technique for obtaining an enriched population of SCs from mature Rhesus monkey nerves, using in vitro pre-degeneration of these nerves in the presence of SC proliferation promoters.

\section{Materials and methods}

Ethics statement. The present study was approved by the ethics committee of Shanghai Jiao Tong University School of Medicine (Shanghai, China). All surgical interventions, treatments and postoperative animal care procedures were performed in accordance with the Guide for the Care and Use of Laboratory Animals. Three adult Rhesus monkeys (4-year-old males, weighing 5.88-8.24 kg) were purchased from Ping'an Animal Reproduction Center of Chengdu (reproduction license no. SCXK 2008-013; Chengdu, China). All monkeys were individually housed at the Department of Laboratory Animal Sciences at Shanghai Jiao Tong University School of Medicine, at a temperature of $21^{\circ} \mathrm{C}$ with $55 \%$ humidity under a 12-h light/dark cycle with free access to food and water.

Materials. Dulbecco's modified Eagle's medium (DMEM) and fetal bovine serum (FBS) were purchased from Hyclone (GE Healthcare Life Sciences, Little Chalfont, UK). Collagenase NB4 was obtained from Serva Electrophoresis $\mathrm{GmbH}$ (Heidelberg, Germany). Neutral protease Dispase II was from Roche Applied Science (Madison, WI, USA). Heregulin- $\beta 1$ and b-FGF were sourced from PeproTech, Inc. (Rocky Hill, NJ, USA). Forskolin was purchased from Cayman Chemical Company (Ann Arbor, MI, USA). Cytosine-B-arabinoside hydrochloride (Ara-C), penicillin-streptomycin and $0.25 \%$ trypsin-EDTA were obtained from Gibco (Thermo Fisher Scientific, Inc., Waltham, MA, USA). All the culture plates were BD Falcon; BD Biosciences (Franklin Lakes, NJ, USA). The compositions of the culture media used for SC isolation are presented in Table I. The following primary antibodies were used for immunofluorescence and flow cytometry: Rabbit anti-S100 calcium binding protein B (S100ß; cat no. Z0311) polyclonal antibody (Dako; Agilent Technologies, Santa Clara, CA, USA), anti-glial fibrillary acidic protein (GFAP;
Table I. Culture media composition.

\begin{tabular}{ll}
\hline Culture medium & \multicolumn{1}{c}{ Composition } \\
\hline Basal medium & DMEM \\
& $10 \% \mathrm{FBS}$ \\
& $1 \%$ penicillin/streptomycin \\
Schwann cell culture medium & DMEM \\
& $10 \% \mathrm{FBS}$ \\
& $1 \%$ penicillin/streptomycin \\
& $2 \mu \mathrm{M}$ forskolin, $20 \mathrm{ng} / \mathrm{ml}$ \\
& heregulin- $\beta 1$ and $20 \mathrm{ng} / \mathrm{ml}$ \\
& b-FGF \\
& DMEM \\
& $2.5 \%$ FBS $/ 10 \mu \mathrm{M}$ Ara-C \\
& $1 \%$ penicillin/streptomycin \\
Purification medium & $2 \mu \mathrm{M}$ forskolin, $20 \mathrm{ng} / \mathrm{ml}$ \\
& heregulin- $\beta 1$ and $20 \mathrm{ng} / \mathrm{ml}$ \\
& b-FGF
\end{tabular}

DMEM, Dulbecco's modified Eagle's medium; FBS, fetal bovine serum; Ara-C, cytosine arabinoside; b-FGF, basic fibroblast growth factor.

cat no. ab7260) polyclonal antibody and anti-nerve growth factor receptor (P75NTR; cat no. ab8874) polyclonal antibody (Abcam, Cambridge, UK). The Alexa Fluor 488-conjugated goat anti-rabbit-IgG secondary antibody (cat no. R37116) was purchased from Invitrogen (Thermo Fisher Scientific, Inc.).

Harvesting of common peroneal nerve and in vitro pre-degeneration. All surgical procedures were performed under sterile surgical conditions and general anesthesia. The animals were anesthetized by intramuscular injection of a mixture of ketamine $(20 \mathrm{mg} / \mathrm{kg})$ and xylazine $(2 \mathrm{mg} / \mathrm{kg})$. Briefly, the bilateral common peroneal nerve was exposed with an incision that extended from the lower interface along the biceps femoris distally towards the popliteal fossa. Two 7-cm segments of the common peroneal nerves were then excised. The nerves were immediately washed three times with ice-cold phosphate-buffered saline (PBS, pH 7.2) supplemented with $2 \%$ FBS. The epineurium was gently stripped off using fine-pointed forceps and microscissors under an anatomical microscope. The common peroneal nerve segments were cut into short pieces (2-3 mm in length), placed into one 6-well plate and cultured first in basal medium (BM) for $3 \mathrm{~h}$ and then switched to Schwann cell culture medium (SCCM). The cells were kept in a humidified atmosphere of $5 \% \mathrm{CO}_{2}$ at $37^{\circ} \mathrm{C}$. The nerve pieces were monitored for evidence of in vitro pre-degeneration on the $2 \mathrm{nd}$, 5th and 7 th day of culture. The finalized process of $\mathrm{SC}$ isolation and purification is summarized and presented in Fig. 1.

Primary culture. For dissociation of the nerve pieces, the pre-degeneration medium was discarded, and the nerve fragments were transferred to a $15-\mathrm{ml}$ conical tube containing $0.2 \%(0.27 \mathrm{U} / \mathrm{ml})$ collagenase NB4 in DMEM and incubated 


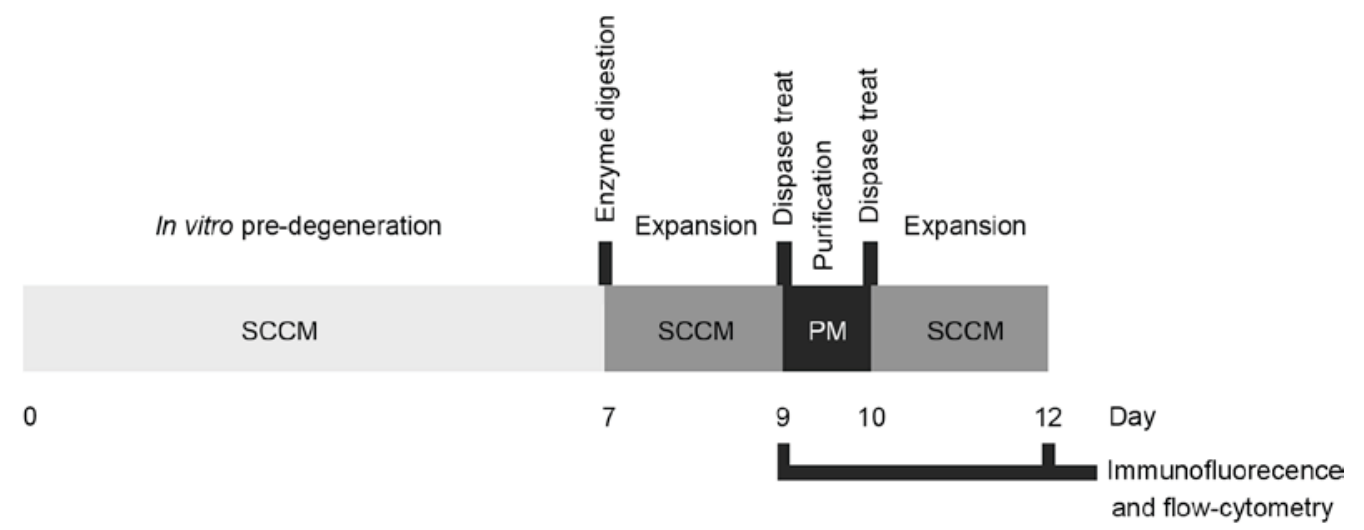

Figure 1. SC isolation and purification process. After a 7-day in vitro pre-degeneration period in SCCM, the nerve pieces were treated enzymatically to dissociate cells, which were cultured for 2 days in SCCM. Subsequently, SCs underwent the first round of purification and were cultured in PM to promote SC proliferation and reduce fibroblast contamination. Following a further $24 \mathrm{~h}$ in PM, SCs underwent a second purification by treatment with a composite encapsulated with collagenase. Finally, the cells were incubated in SCCM. SC, Schwann cell; SCCM, SC culture medium; PM, purification medium.

at $37^{\circ} \mathrm{C}$ for $45-60 \mathrm{~min}$ until the cells were dispersed into a homogeneous suspension. Following centrifugation of the suspension at $600 \mathrm{x} \mathrm{g}$ for $5 \mathrm{~min}$ at room temperature, the supernatant was removed and discarded, and the cells were re-suspended in SCCM. The cells were subsequently seeded onto one laminin-coated six-well plate and cultivated at $37^{\circ} \mathrm{C}$ in $5 \% \mathrm{CO}_{2}$ for $48 \mathrm{~h}$ prior to isolation of the SCs.

Purification of SCs. Differential detachment methods were applied as previously described (10). Following cultivation in the six-well plate for $48 \mathrm{~h}$, the SCCM was replaced with $0.1 \mathrm{ml} / \mathrm{cm}^{2}$ of Dispase II $(1.25 \mathrm{U} / \mathrm{ml})$ diluted in DMEM. The cells were then incubated at $37^{\circ} \mathrm{C}$ for $10-15 \mathrm{~min}$ and subsequently shaken horizontally for $1 \mathrm{~min}$ to release rounded-up cells. The suspended cells were transferred to a $15-\mathrm{ml}$ conical tube and centrifuged at $600 \mathrm{x}$ g for $5 \mathrm{~min}$ at room temperature. The supernatant was discarded, and the cells were re-suspended in purification medium (PM) to reduce the fibroblast contamination, and cultured for $24 \mathrm{~h}$. After another 24-h cultivation period, the cells were subjected once more to the aforementioned purification steps (Dispase II treatment) and re-suspended in SCCM (Fig. 1).

Immunostaining of $S 100 \beta, P 75 N T R$ and GFAP. In order to characterize the isolated cells, S100 $\beta$, P75NTR and GFAP immunostaining was performed. The cells were fixed to slides with $4 \%$ paraformaldehyde (PFA) for $15 \mathrm{~min}$ at room temperature, washed three times with PBS, treated with $0.3 \%$ Triton $\mathrm{X}-100$ to permeabilize the membranes, washed again in PBS and blocked with $10 \%$ bovine serum album (Sigma-Aldrich; Merck KGaA) at $37^{\circ} \mathrm{C}$ for $30 \mathrm{~min}$. The slides were subsequently incubated with rabbit anti-S100 polyclonal antibody (1:200), rabbit anti-P75NTR polyclonal antibody (1:500) or rabbit anti-GFAP polyclonal antibody $(1: 500)$ at $37^{\circ} \mathrm{C}$ for $1 \mathrm{~h}$. The slides were washed three times with PBS and incubated with a FITC-conjugated goat anti-rabbit IgG secondary antibody (1:500; cat no. F2765) for $1 \mathrm{~h}$ at $37^{\circ} \mathrm{C}$, and finally incubated with 4', 6'-diamidino-2-phenylindole dihydrochloride (DAPI; 1:500; Invitrogen; Thermo Fisher Scientific, Inc.) for 2 min at room temperature to stain the cell nuclei. Images were captured under a fluorescence microscope (Olympus Corporation, Tokyo,
Japan) and processed using Image-Pro Plus version 6.0 (Media Cybernetics, Inc., Rockville, MD, USA). Cells in three randomly selected fields were counted to calculate the SC purity.

Flow cytometric analysis. To further analyze SC purity using specific marker molecules, P0 (before purification) cells and P2 (after purification) cells were collected after $1 \mathrm{~min}$ incubation with $0.25 \%$ trypsin-EDTA at $37^{\circ} \mathrm{C}$, washed with and suspended in blocking buffer (2\% FBS in PBS) and incubated with rabbit anti-P75NTR antibody (1:500) for $30 \mathrm{~min}$ at $4^{\circ} \mathrm{C}$. The cells were subsequently washed twice with blocking buffer and incubated with an Alexa Fluor 488-conjugated goat anti-rabbit-IgG secondary antibody $(1: 1,000)$ for $15 \mathrm{~min}$ at $4^{\circ} \mathrm{C}$. Cells were analyzed using an EPICS Altra flow cytometer (Beckman Coulter, Inc., Fullerton, CA, USA). Cells without primary antibody treatment were used as a blank control.

SC purity and cell yield. SCs were morphologically identified from fibroblasts under a phase-contrast microscope and counted in three randomly selected fields to obtain an average number. Cells with a bipolar or tripolar shape were identified as SCs and flat or polygonal cells were identified as fibroblasts. Following trypsinization, all cells (SCs and fibroblasts) were counted with a hemocytometer to determine the total yield. Purity was defined as the percentage of SCs relative to the total number of counted cells. Cell yields were expressed as the mean of $10^{6}$ cells per biopsy. Cell number was presented as the mean \pm standard deviation (SD).

Statistical analysis. Data were presented as the mean values \pm SD. For quantitative comparison and analysis, an unpaired Student's t-test and one way analysis of variance followed by Dunnett's post hoc test were used to compare differences between groups. All statistical analyses were performed using SPSS version 18.0 (SPSS, Inc., Chicago, IL, USA). P-values $<0.05$ were considered statistically significant.

\section{Results}

Cell yield and SC purity following in vitro pre-degeneration. Following 2 days of pre-degeneration treatment, several 
A
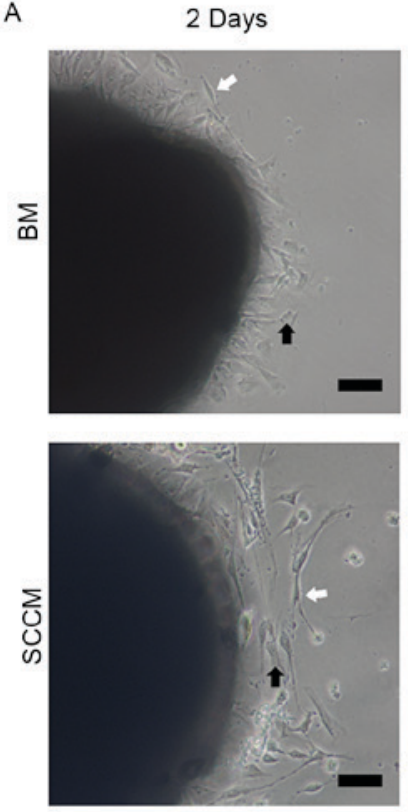

5 Days
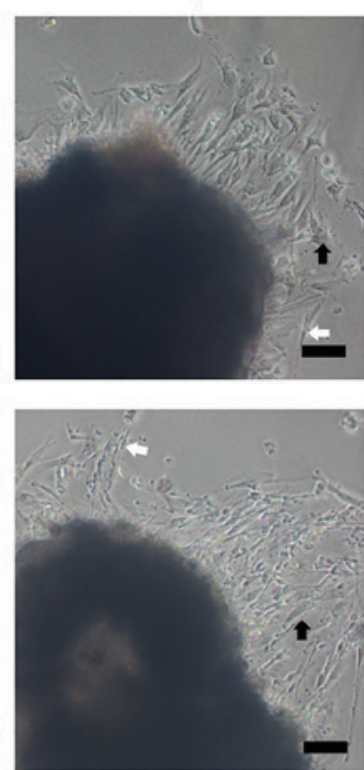

7 Days
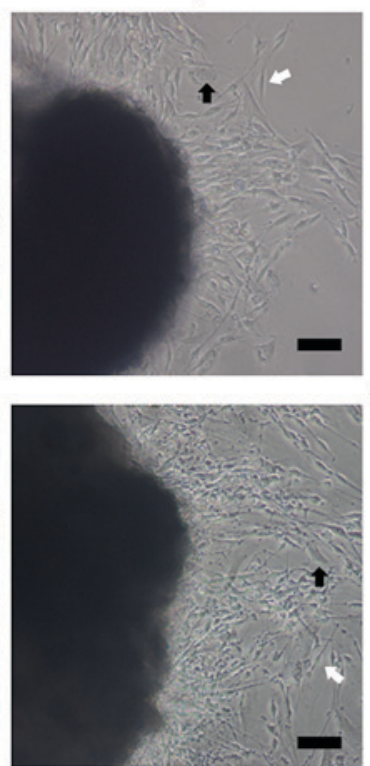

B
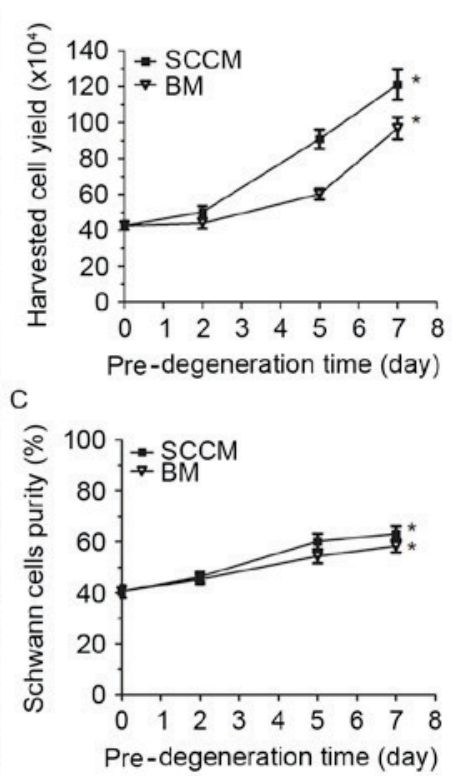

Figure 2. SC purity and cell yield following in vitro pre-degeneration at various time points in BM and SCCM. (A) SCs (white arrow) and fibroblasts (black arrow) were observed to migrate out of the nerve explants in BM and SCCM. Nerve tissue is evident on the left. Scale bars $=100 \mu \mathrm{m}$. An improvement in (B) SC yield (from $50 \pm 8.2 \times 10^{4}$ to $120 \pm 21 \times 10^{4}$ cells/well) and (C) SC purity (from $40.50 \pm 5.8$ to $63.17 \pm 7.1 \%$ ) was observed following 7 days of pre-degeneration in SCCM. Pre-degeneration in BM induced an increased in SC yield from $50 \pm 8.2 \times 10^{4}$ to $96 \pm 1.5 \times 10^{5}$ cells/well and SC purity from $40.50 \pm 5.8$ to $58.33 \pm 6.2 \%$. ${ }^{*} \mathrm{P}<0.05$. SC, Schwann cell; SCCM, SC culture medium; BM, basal medium.

cells began to migrate out of the nerve explants and attach to the culture dish. The number of cells that migrated out of the nerve explants increased with increased treatment time. The cells migrating into the BM were predominantly fibroblasts and rarely SCs (Fig. 2A). Following 2, 5 and 7 days of pre-degeneration, the nerve explants were subjected to enzymatic digestion and examined to determine SC yield prior to seeding the cells onto laminin-coated six-well plates. The cell yield (Fig. 2 B) was $50 \pm 8.2 \times 10^{4}$ cells/well after 2 days of pre-degeneration in SCCM, and $44 \pm 7.2 \times 10^{4}$ cells/well after 2 days of pre-degeneration in BM. The cell yield in SCCM $\left(120 \pm 21 \times 10^{4}\right.$ cells/well) was 1.25 -fold higher compared with the BM media $\left(96 \pm 1.5 \times 10^{4}\right.$ cells/well) after 7 days of pre-degeneration, and was 2.84 times higher compared with the immediate culture cells that had not undergone pre-degeneration $\left(43 \pm 1.5 \times 10^{4}\right.$ cells/well). In addition, the purity of the SCs was slightly higher after 7 days of pre-degeneration and culture in SCCM $(63.17 \pm 7.1 \%)$ compared with 7 days of

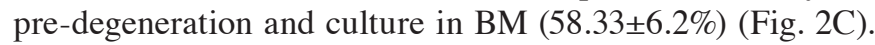
These results demonstrated that SC purity and yield were greater than the fibroblast purity and yield following in vitro pre-degeneration in SCCM.

Primary culture and phase-contrast photomicrographs of SCs. The majority of cells suspended in SCCM adhered to the laminin-coated flasks within $24 \mathrm{~h}$ and exhibited two distinct morphologies: SCs and fibroblasts (Fig. 3A). Fibroblasts were characterized by a regular polygonal shape and ovoid nucleus (Fig. 3B). The SCs were characterized as small, elongated bipolar (Fig. 3C), tripolar (Fig. 3D) or multipolar (Fig. 3E) cells with a bright nucleus. The fibroblasts were tightly attached to the cell plate and adhered under the SCs (Fig. 3F), which facilitated the removal of the SCs.

Cell yield and SC purity following purification. Following the first dispase treatment, the SCs appeared to proliferate faster than the fibroblasts (Fig. 4A); however, the fibroblasts subsequently became the predominant cell type. Therefore, Ara-C was used to inhibit fibroblast growth during the 48-72 h period. Following the first round of SC purification on day 9 and the use of Ara-C (Fig. 4B), SC purity markedly increased to $85.1 \pm 7.4 \%$, determined by cell counting based on morphological differences. However, the remaining fibroblasts continued to proliferate rapidly and an additional round of purification was performed on day 10. Following the second round of purification and subsequent $48 \mathrm{~h}$ culture, SC purity reached $96.3 \pm 3.9 \%$ (Fig. 4C and D). A significant difference in SC purity was observed between days 9 and 12. To determine the final cell yield, the enriched SCs were identified by cell counting using a hematocytometer on days 9, 10 and 12. The cell yield increased and reached $2.1 \pm 0.2 \times 10^{6}$ cells/well following the second round of purification (Fig. 4E). These results demonstrated that cell yield and SC purity were significantly increased following two rounds of purification.

Immunostaining of SC markers. Immunostaining for $\mathrm{S} 100 \beta$ protein (predominantly expressed in SC nuclei), P75NTR (primarily expressed on the cell surface and may be used as a marker in flow cytometric analysis) and GFAP (expressed in cells lacking fibronectin) were used to differentiate SCs from fibroblasts. Purified SCs exhibited typical bipolar or tripolar morphology and oval nuclei and 

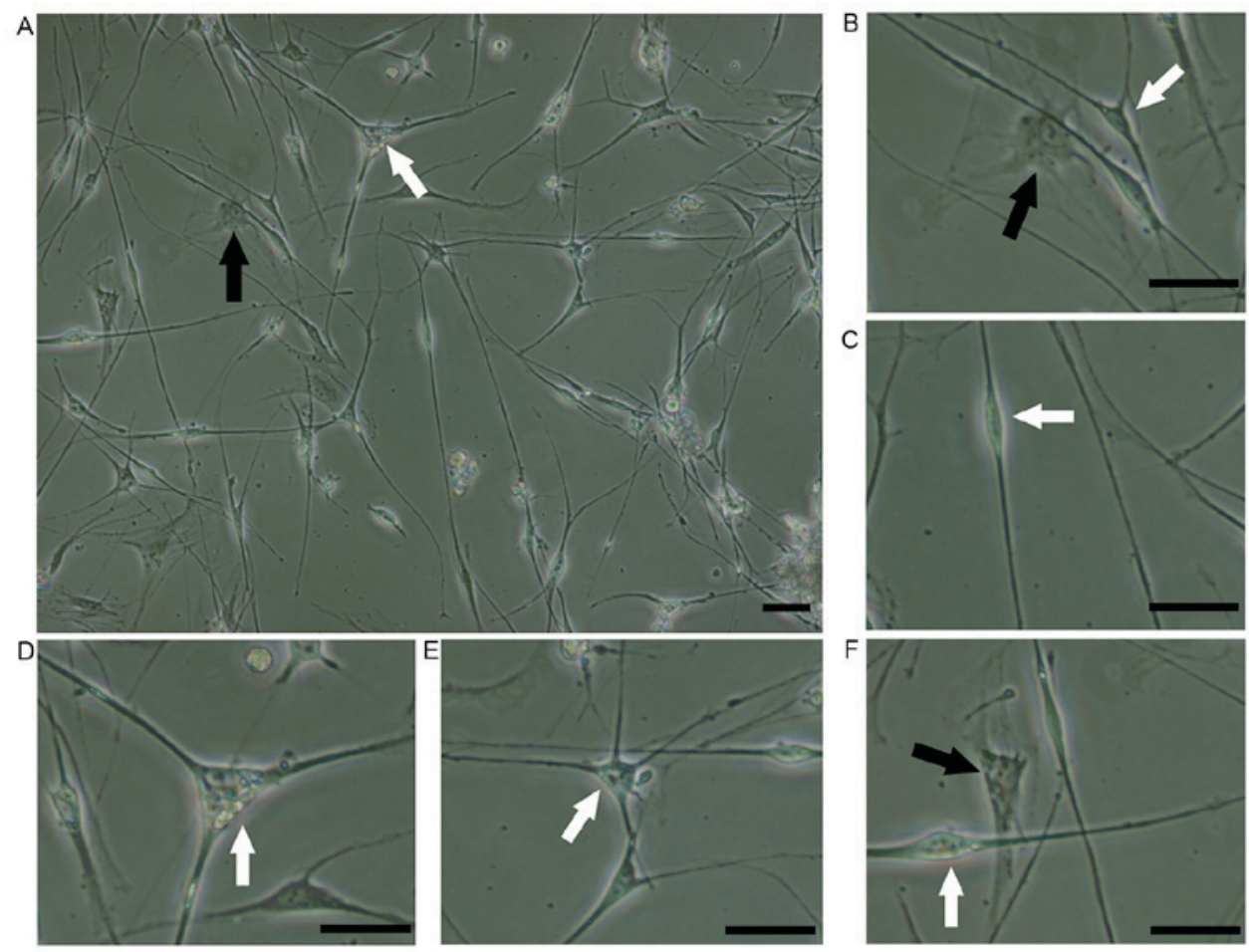

Figure 3. Cell morphology following $24 \mathrm{~h}$ of primary culture. (A) Majority of cells adhered to laminin-coated flasks and had two different cell morphologies: SCs and fibroblasts. (B) Fibroblasts exhibited a polygonal shape and were observed growing among the SCs. SCs were observed with a (C) bipolar, (D) tripolar or (E) multipolar shape. (F) Fibroblasts were observed growing under the layer of SCs. Black arrows, fibroblasts; white arrows, SCs. Scale bars=50 $\mu \mathrm{m}$. SC, Schwann cell.
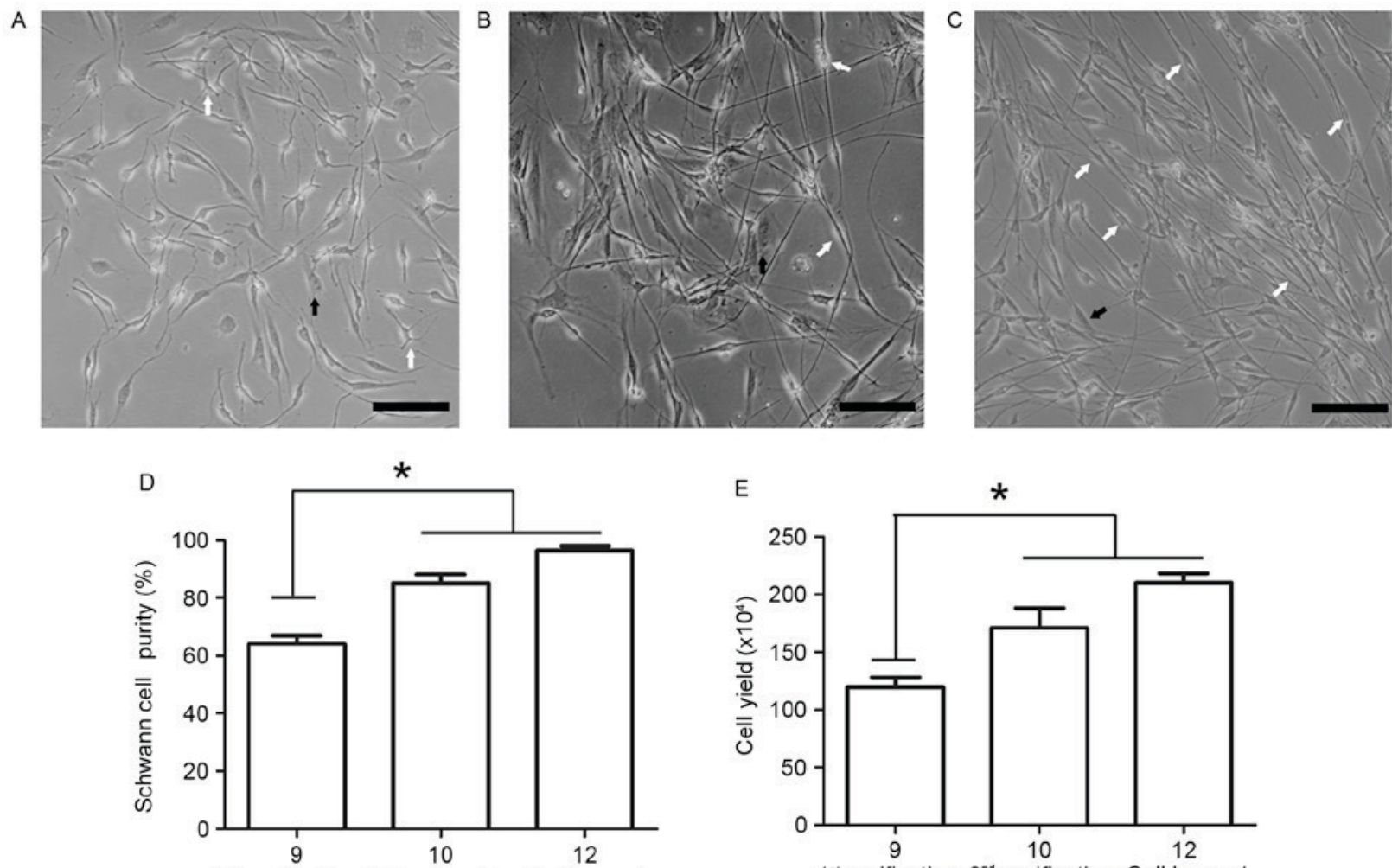

$1^{\text {tst }}$ purification $2^{\text {nd }}$ purification Cell harvest Culture time (days)

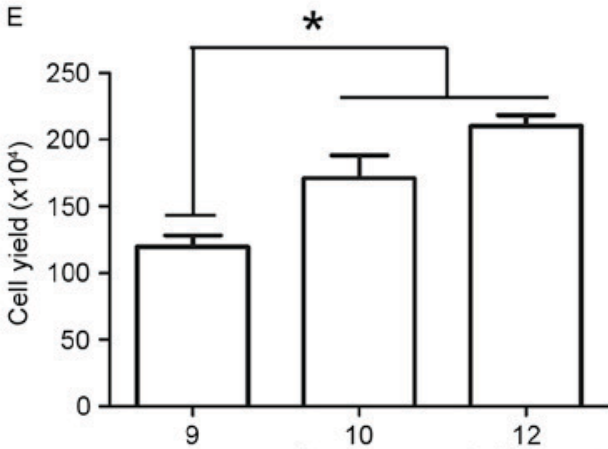

$1^{\text {st }}$ purification $2^{\text {nd }}$ purification Cell harvest

Culture time (days)

Figure 4. Cell yield and SC purity following purification. (A) Majority cells were observed to adhere to the plates following $24 \mathrm{~h}$ of primary culture. (B) Following treatment with purification medium, fibroblast cell numbers decreased, whereas SC purity increased. (C) SC purity was increased again following the second round of purification. Black arrows, fibroblasts; white arrows, SCs. Scale bars $=100 \mu \mathrm{m}$. (D) SC purity at various time points of the SC purification procedure. (E) Cell yield at various time points of the SC purification procedure. A significant difference in SC purity was observed between days 9 and 12 . " $\mathrm{P}<0.05$, as indicated. SC, Schwann cell. 


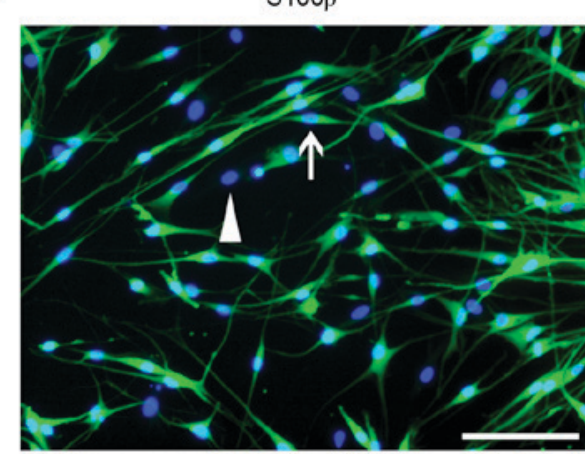

C

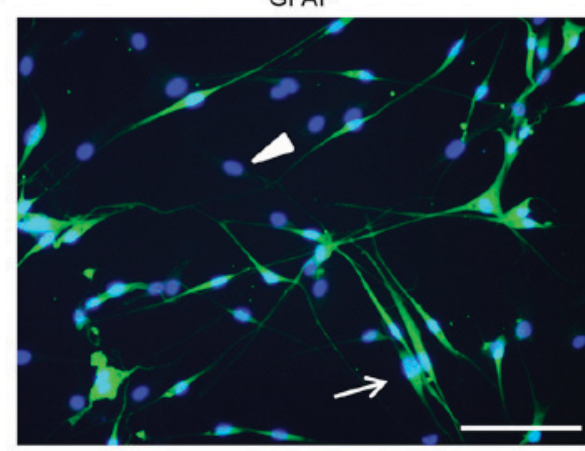

B

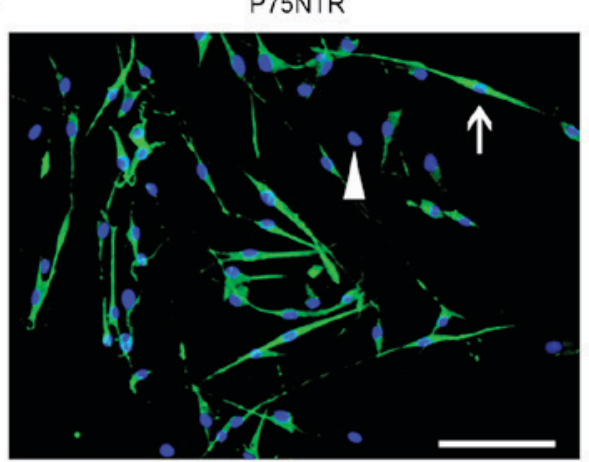

D

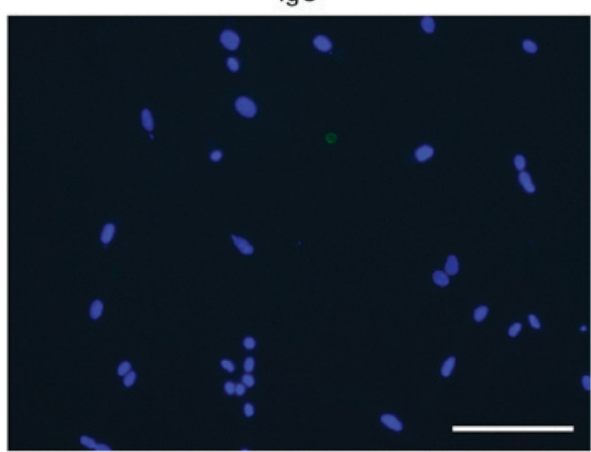

Figure 5. Immunofluorescence staining for S100 $\beta$, P75NTR and GFAP. All SCs with bipolar or tripolar morphology (compared with the flat fibroblasts) were positive for (A) S100ß, (B) P75NTR and (C) GFAP. Nuclei were visualized by DAPI staining. (D) Non-specific staining (green) with secondary antibody control (rabbit IgG). White arrow head, fibroblasts; white arrow, SCs. Scale bars=100 $\mu \mathrm{m}$. SC, Schwann cell; S100 $\beta$, S100 calcium binding protein B; P75NTR, nerve growth factor receptor; GFAP, glial fibrillary acidic protein.

unlike fibroblasts, were immunopositive for S100ß,P75NTR and GFAP (Fig. 5).

Flow cytometric analysis. To provide further quantitative evidence of SC purity, the cells were analyzed before and after purification by flow cytometry with a P75NTR-specific rabbit antibody and tagged with an anti-rabbit IgG antibody (Alexa Fluor 488). Following pre-degeneration, cell suspensions obtained from collagenase digestion of the nerve explants were incubated for $48 \mathrm{~h}$, immunostained for P75NTR, and analyzed by flow cytometry. The percentage of P75NTR-positive SCs was $65.2 \pm 1.3 \%$ at $48 \mathrm{~h}$ and $98.4 \pm 1.5 \%$ following one purification cycle. There was a significant difference in cell percentages (Fig. 6).

\section{Discussion}

The SC-coated scaffold is an effective strategy to repair peripheral nerve defects (17). Therefore, populations of highly purified SCs may be needed in sufficient quantity and on short notice. Researchers have investigated a variety of methods to satisfy this requirement, including repeated explantation methods (8), differential adhesion and detachment methods (18), immunoselective methods (19) and serum tapering (20), amongst others. Although they are effective at purifying SCs, these methods have limitations $(10,21,22)$, including the requirement for special equipment, complicated procedures and high cost.

Experimentally, embryonic or newborn mice and rats are currently the predominant source of non-myelinating SCs, which have a strong capacity to proliferate (23). However, in most treatment strategies, SCs are obtained from adult animals. Casella et al $(24,25)$ demonstrated that the culture and amplification of SC populations becomes more difficult as the age of the animal increases. Therefore, the development of a method for culturing SCs from adult animals may be clinically more relevant as nerve injury frequently occurs in adults. Monkeys are ideal models for studying diseases of the human nervous system. Newborn and adult monkeys have fully mature neural structures with nerve bundles surrounded by epineurium; however, this makes SC migration from nerve explants difficult. In the present study, pre-degeneration of the common peroneal nerve in vitro resulted in SC proliferation and migration. The SCs were subsequently enzymatically dissociated from the nerve pieces and purified. Cell yield and SC purity were increased following nerve explant pre-degeneration in vitro in the presence of mitogen-activated protein (heregulin- $\beta 1$ ) and an activator of adenylate cyclase (forskolin).

During in vivo pre-degeneration, SCs dedifferentiate and proliferate following peripheral nerve transaction (20). A period of time must pass in order to allow for Wallerian degeneration to occur and SCs can subsequently be enzymatically dissociated from the nerve. In vivo pre-degeneration procedures are cumbersome to perform, and the requirement for two independent surgical procedures means this process is not ethically acceptable in human research. Therefore, the present study investigated the in vitro approach. Different pre-degeneration conditions may impact upon the yield and purity of SCs (10). Kraus et al (13) compared SC yield and purity after 2, 7 and 14 days of in vitro pre-degeneration, 

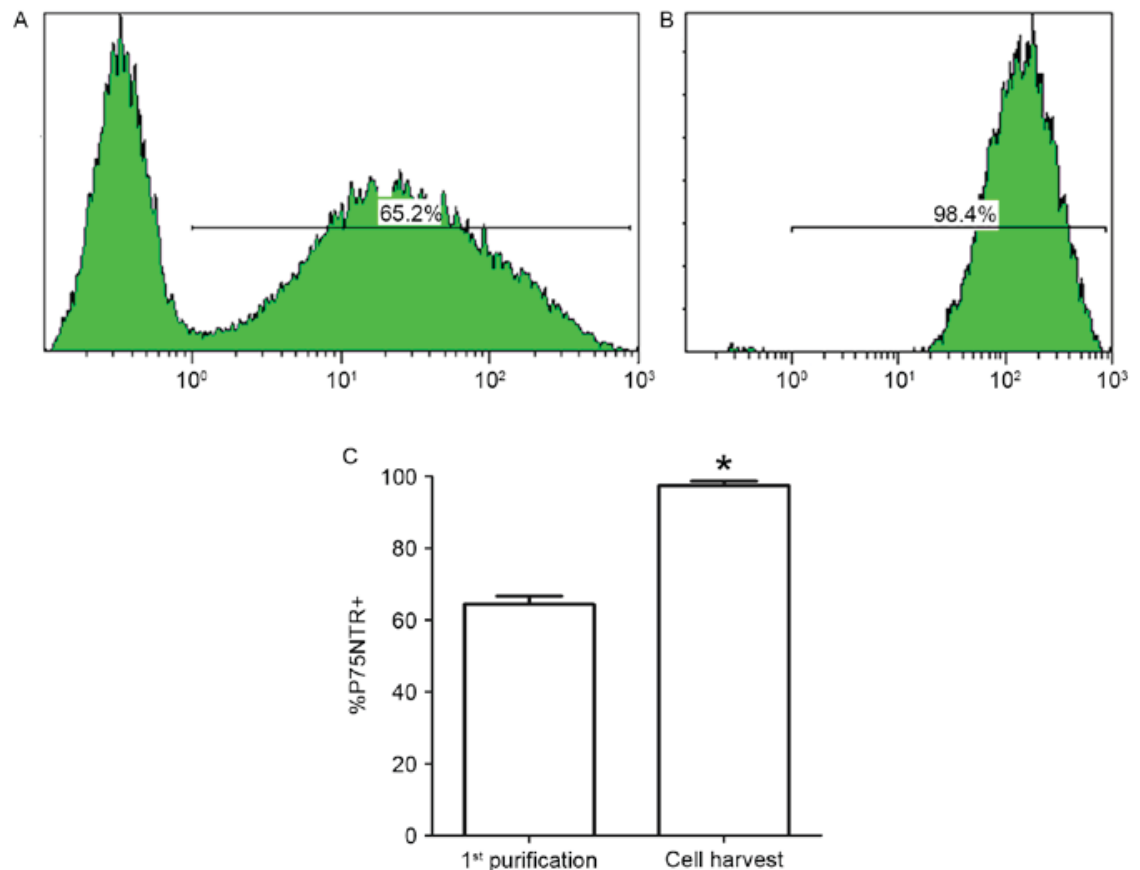

Figure 6. FCM analysis of P75NTR-positive SCs. P75NTR-positive SCs (A) prior to and (B) following purification. FCM analysis demonstrated an increase in the purity of cells expressing P75NTR protein from $65.2 \pm 1.3$ to $98.4 \pm 1.5 \%$ following purification. (C) Bar graph presentation of the quantified data. ${ }^{*} \mathrm{P}<0.05$ vs. 1st purification. FCM, flow-cytometric; P75NTR, nerve growth factor receptor; SC Schwann cells.

and demonstrated a doubling of the yield after 7 days when compared with 2 days. It is of note that marked contamination of the culture by fibroblasts was observed after longer pre-degeneration periods. Therefore, they recommended the 7-day pre-degeneration period, which is in accordance with the method used in the present study. Levi et al (22) demonstrated that heregulin- $\beta$ stimulates the heregulin receptors erb-b2 receptor tyrosine kinase (erbB) 2 and erbB3 in SCs and leads to the activation of the mitogen activated protein kinase pathway, and demonstrated that forskolin significantly enhances the expression level of a group of genes associated with cell division. SC proliferation requires long-term simultaneous exposure to heregulin plus forskolin as well as bFGF (an independent activator of the extracellular signal-related kinase pathway, which is important for SC proliferation) (26).

In the present study, in vitro pre-degeneration was performed in SCCM or BM. Following pre-degeneration for 7 days, the cell yield was 1.25 times higher in SCCM compared with BM, and 2.84 times higher in SCCM compared with non-pre-degeneration conditions. SC purity was also markedly higher in SCCM compared with non-pre-degeneration conditions. Based on the present observations, the migration rate of SCs out of the nerve explants appeared to reach its maximum after 14 days (data not shown). However, the level of fibroblast proliferation was also significantly increased, which reduced the purity of the SCs. Therefore, the shorter 7-day pre-degeneration period may be optimal to maximize SC purity.

The primary obstacle to SC purification is fibroblast contamination. Anti-mitotic and low-serum treatments at the appropriate time may be an effective method to inhibit fibroblast proliferation. The present study developed several strategies to reduce fibroblast contamination: i) Removal of the epineurium as completely as possible; ii) application of $\mathrm{PM}$ at the appropriate time, in this study this was after $48 \mathrm{~h}$ (the time when the proliferation rate is higher in fibroblasts compared with SCs); and iii) use of differential detachment methods. This series of purification steps increased SC purity from 65.2 to $98.4 \%$.

In conclusion, the newly established protocols in the present study provide a rapid and efficient method for obtaining highly enriched populations of SCs from mature Rhesus monkey nerves. These cells may be used to provide support for regenerating peripheral or central nerves.

\section{Acknowledgements}

The current study was supported by the National Natural Science Foundation (grant nos. 31170932 and 81000522) and the Lanzhou Personnel Entrepreneurship and Innovation Project (grant no. 2015-RC-74). The animal studies were performed in the Department of Laboratory Animal Science, Shanghai Jiaotong University School of Medicine. The authors would like to thank Norton Healthcare (Louisville, KY, USA) for their ongoing support. We sincerely thank Mr Weihua Lu (Shanghai Jiao Tong University, Shanghai, China) for his commitment to animal care.

\section{References}

1. Fawcett JW and Keynes RJ: Peripheral nerve regeneration. Annu Rev Neurosci 13: 43-60, 1990.

2. Dezawa M: Central and peripheral nerve regeneration by transplantation of Schwann cells and transdifferentiated bone marrow stromal cells. Anat Sci Int 77: 12-25, 2002.

3. Madduri S and Gander B: Schwann cell delivery of neurotrophic factors for peripheral nerve regeneration. J Peripher Nerv Syst 15: 93-103, 2010 
4. Yang XN, Jin YQ, Bi H, Wei W, Cheng J, Liu ZY, Shen Z, Qi ZL and Cao Y: Peripheral nerve repair with epimysium conduit. Biomaterials 34: 5606-5616, 2013.

5. Hedayatpour A, Sobhani A, Bayati V, Abdolvahhabi MA Shokrgozar MA and Barbarestani M: A method for isolation and cultivation of adult Schwann cells for nerve conduit. Arch Iran Med 10: 474-480, 2007.

6. Needham LK, Tennekoon GI and McKhann GM: Selective growth of rat Schwann cells in neuron- and serum-free primary culture. J Neurosci 7: 1-9, 1987.

7. Rutkowski JL, Kirk CJ, Lerner MA and Tennekoon GI: Purification and expansion of human Schwann cells in vitro. Nat Med 1: 80-83, 1995.

8. Pannunzio ME, Jou IM, Long A, Wind TC, Beck G and Balian G: A new method of selecting Schwann cells from adult mouse sciatic nerve. J Neurosci Methods 149: 74-81, 2005.

9. Jin YQ, Liu W, Hong TH and Cao Y: Efficient Schwann cell purification by differential cell detachment using multiplex collagenase treatment. J Neurosci Methods 170: 140-148, 2008.

10. Mauritz C, Grothe $C$ and Haastert K: Comparative study of cell culture and purification methods to obtain highly enriched cultures of proliferating adult rat Schwann cells. J Neurosci Res 77: 453-461, 2004.

11. Haastert K, Mauritz C, Matthies C and Grothe C: Autologous adult human Schwann cells genetically modified to provide alternative cellular transplants in peripheral nerve regeneration. J Neurosurg 104: 778-786, 2006.

12. Dreesmann L, Mittnacht U, Lietz M and Schlosshauer B: Nerve fibroblast impact on Schwann cell behavior. Eur J Cell Biol 88: 285-300, 2009.

13. Kraus A, Tager J, Kohler K, Manoli T, Haerle M, Werdin F, Hoffmann J, Schaller HE and Sinis N: Efficacy of various durations of in vitro predegeneration on the cell count and purity of rat Schwann-cell cultures. J Neurotrauma 27: 197-203, 2010.

14. Haastert K, Seef P, Stein VM, Tipold A and Grothe C: A new cell culture protocol for enrichment and genetic modification of adult canine Schwann cells suitable for peripheral nerve tissue engineering. Res Vet Sci 87: 140-142, 2009.

15. Rahmatullah M, Schroering A, Rothblum K, Stahl RC, Urban B and Carey DJ: Synergistic regulation of Schwann cell proliferation by heregulin and forskolin. Mol Cell Biol 18: 6245-6252, 1998.
16. Zhu J, Qin J, Shen Z, Kretlow JD, Wang X, Liu Z and Jin Y: Dispase rapidly and effectively purifies Schwann cells from newborn mice and adult rats. Neural Regen Res 7: 256-260, 2012.

17. Wang G, Cao L, Wang Y, Hua Y, Cai Z, Chen J, Chen L, Jin Y, Niu L, Shen H, et al: Human eyelid adipose tissue-derived Schwann cells promote regeneration of a transected sciatic nerve. Sci Rep 7: 43248, 2017.

18. Wei Y, Zhou J, Zheng Z, Wang A, Ao Q, Gong Y and Zhang X: An improved method for isolating Schwann cells from postnatal rat sciatic nerves. Cell Tissue Res 337: 361-369, 2009.

19. Manent J, Oguievetskaia K, Bayer J, Ratner N and Giovannini M: Magnetic cell sorting for enriching Schwann cells from adult mouse peripheral nerves. J Neurosci Methods 123: 167-173, 2003.

20. Kang SH, Shin GW, Shin YS, J PK, Kim YR, Yang HH, Lee EY, Lee EG, Huh NE, Ju OM and Jung TS: Experimental evaluation of pathogenicity of Lactococcus garvieae in black rockfish (Sebastes schlegeli). J Vet Sci 5: 387-390, 2004.

21. Tomita K, Hata Y, Kubo T, Fujiwara T, Yano K and Hosokawa K: Effects of the in vivo predegenerated nerve graft on early Schwann cell migration: Quantitative analysis using S100-GFP mice. Neurosci Lett 461: 36-40, 2009.

22. Levi AD, Bunge RP, Lofgren JA, Meima L, Hefti F, Nikolics K and Sliwkowski MX: The influence of heregulins on human Schwann cell proliferation. J Neurosci 15: 1329-1340, 1995.

23. Niapour N, Mohammadi-Ghalehbin B, Golmohammadi MG, Gholami MR, Amani M and Niapour A: An efficient system for selection and culture of Schwann cells from adult rat peripheral nerves. Cytotechnology 68: 629-636, 2016.

24. Casella GT, Bunge RP and Wood PM: Improved method for harvesting human Schwann cells from mature peripheral nerve and expansion in vitro. Glia 17: 327-338, 1996.

25. Casella GT, Wieser R, Bunge RP, Margitich IS, Katz J, Olson L and Wood PM: Density dependent regulation of human Schwann cell proliferation. Glia 30: 165-177, 2000.

26. Schworer CM, Masker KK, Wood GC and Carey DJ: Microarray analysis of gene expression in proliferating Schwann cells: Synergistic response of a specific subset of genes to the mitogenic action of heregulin plus forskolin. J Neurosci Res 73: 456-464, 2003. 